\title{
EVIDENCIAS TECNOLÓGICAS EN ASTAS DE VENADO DE LAS PAMPAS EN EL CENTRO DE LA PAMPA HÚMEDA, ARGENTINA
}

\author{
TECHNOLOGICAL EVIDENCE IN PAMPAS DEER ANTLERS FROM THE \\ CENTER OF THE HUMID PAMPAS, ARGENTINA
}

\author{
María Clara Álvarez ${ }^{1}$
}

\begin{abstract}
En los sitios del centro de la Pampa Húmeda argentina es frecuente el hallazgo de restos de asta de venado de las Pampas (Ozotoceros bezoarticus). Sin embargo, estos materiales suelen registrarse en escasas cantidades y, salvo excepciones, sólo son mencionados colateralmente en la literatura arqueológica. En este trabajo se presenta el análisis de los restos de astas de venado de las Pampas de dos sitios arqueológicos: Empalme Querandíes 1 y Calera. Los resultados indican que estos elementos fueron utilizados con fines tecnológicos. Sin embargo, algunas partes de las astas (las puntas) no serían las más diagnósticas para identificar este comportamiento. Esto es porque gran parte de las modificaciones que se clasifican como culturales también se producen en vida del animal. Por otro lado, las bases presentan evidencias de abrasión y negativos de lascado, que serían buenos indicadores del uso de este elemento como instrumento. En este estudio se identificaron varias bases con modificaciones, que podrían haber sido utilizadas como percutores blandos. Finalmente, la técnica utilizada para la obtención de las formas base habría sido la de débitage por segmentación.
\end{abstract}

Palabras claves: tecnología ósea, venado de las Pampas, asta, región pampeana.

Antler remains of Pampas deer (Ozotoceros bezoarticus) are frequently recorded in the sites located in the center of the Argentinian Humid Pampas. These materials, however, are usually scarce and, in general, they are only collaterally mentioned in the archaeological literature. This article presents the analysis of antler remains from two archaeological sites -Empalme Querandies 1 and Calera-. Results indicate that these elements were used with technological purposes. However, some parts of the antler (the tines) are not the more diagnostic ones to identify this behavior. This is because a large part of the modifications that are classified as "of cultural origin" also occur in the animal's life. The bases, on the other hand, can present evidence of abrasion and flake negatives, which could be good indicators of the use of these elements as tools. In this study, several specimens with modifications, which could have been used as soft hammers, were identified. Finally, the technique that was used in the obtaining of blanks would have been débitage by segmentation.

Key words: Bone technology, Pampas deer, antler, Pampas region.

El centro de la subregión Pampa Húmeda de Argentina (Figura 1) ha sido objeto de investigaciones arqueológicas desde hace varias décadas. Las sociedades que habitaron esta área eran cazadoresrecolectores móviles, que basaron su subsistencia en el consumo de guanaco (Lama guanicoe) (Politis 2008). La mayoría de los estudios sobre estos grupos se ha focalizado en aspectos tales como los circuitos de movilidad, la subsistencia o la tecnología cerámica y lítica. Sin embargo, en años recientes, se ha comenzado a prestar mayor atención a la tecnología ósea (Álvarez 2012; Frontini et al. 2017), que fue relegada principalmente porque este registro no es abundante ni estandarizado. En general y salvo excepciones, los artefactos presentan una elaboración escasa y no se registran desechos de la manufactura (Álvarez 2012). La obtención de los soportes para confeccionar instrumentos estaría vinculada con el procesamiento de los animales para su consumo (Mazzanti y Valverde 2001).

\footnotetext{
${ }^{1}$ INCUAPA-CONICET (Investigaciones Arqueológicas y Paleontológicas del Cuaternario Pampeano), Facultad de Ciencias Sociales, Universidad Nacional del Centro de la Provincia de Buenos Aires, Avenida del Valle 5737, B7400JWI Olavarría, Buenos Aires, Argentina. malvarez@soc.unicen.edu.ar
} 


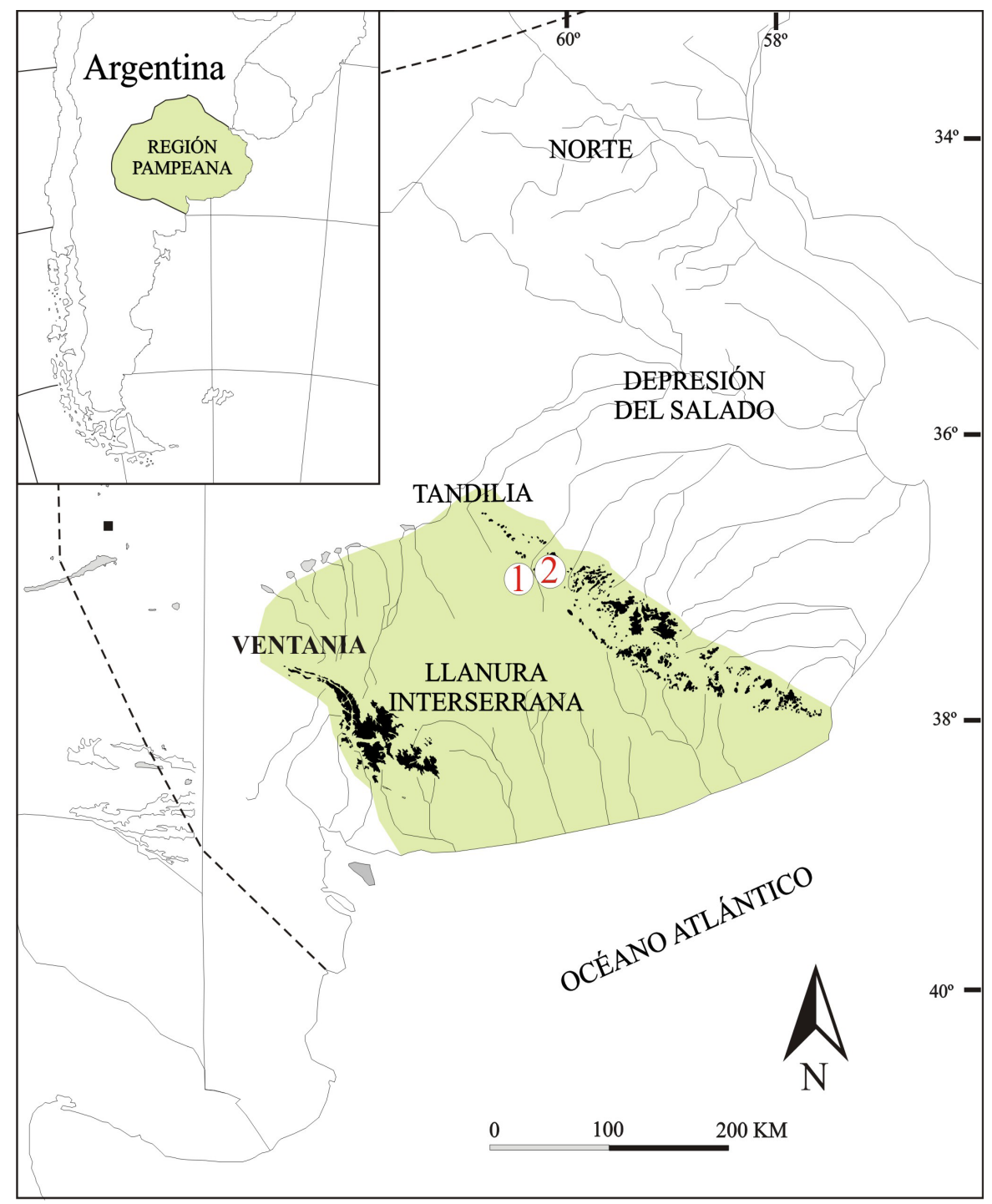

Figura 1. Región pampeana argentina y ubicación de los sitios Empalme Querandíes 1 (1) y Calera (2) en el centro de la Pampa Húmeda. Argentinian Pampas region and location of the sites Empalme Querandies 1 (1) and Calera (2) in the center of the Humid Pampas. 
El uso del asta como materia prima es habitual en la región pampeana, aunque varía en las diferentes áreas de la misma. Si bien los restos de cérvidos son frecuentemente recuperados en los sitios arqueológicos, generalmente constituyeron un recurso principalmente alimenticio (Martínez y Gutiérrez 2004). Sin embargo, en algunas partes de la región, como el Delta del río Paraná, las cornamentas de estos animales fueron comúnmente utilizadas para la fabricación de instrumentos, como por ejemplo las puntas cónicas, ahuecadas o cabezales de arpones (p.ej., Acosta et al. 2014; Bonomo 2013; Buc y Loponte 2007; Politis y Bonomo 2015). En otros sectores, como la Depresión del Salado, se ha propuesto el uso de los restos de asta como instrumentos, sin modificaciones previas, aunque su registro es mucho más escaso (Escosteguy et al. 2017).

La única especie nativa de cérvido que habitó el centro de la Pampa Húmeda argentina durante el Holoceno fue el venado de las Pampas (Ozotoceros bezoarticus). Este animal formó parte de la dieta humana y su explotación ha sido reconocida en una amplia cantidad de sitios (Politis et al. 2011). En esta área, los fragmentos de cornamentas se registran ocasionalmente en los conjuntos arqueofaunísticos. Aunque son escasos, estos materiales están presentes en varios sitios con diferentes cronologías, tales como Arroyo Seco 2, Alfar, Cueva Tixi, Paso Otero 3, Paso Otero 4, La Toma y Cortaderas, entre otros (Álvarez 2012; Bonomo y León 2010; Martínez et al. 2001; Massigoge 2009; Mazzanti y Valverde 2001; Salemme 2014). En general, los especímenes recuperados corresponden a fragmentos de las puntas de las cornamentas y algunas de ellas han sido determinadas como instrumentos o retocadores, debido a sus superficies pulidas y la presencia de marcas (Bonomo y León 2010; Kaufmann y Álvarez 2007; Mazzanti y Valverde 2001). Dado que las astas son tejidos duros pero a la vez flexibles, constituyen excelentes materiales para realizar actividades relacionadas con la talla lítica (Currey 1984; Guthrie 1983; Jin y Shipman 2010). En estas tareas suelen utilizarse percutores de piedra para la reducción inicial, pero también percutores blandos, como las bases de las astas, para las extracciones más controladas, y las puntas de las cornamentas para realizar lascados por presión (Bello et al. 2016; Nami y Scheinsohn 1997).

El objetivo de este trabajo es determinar el aprovechamiento de las diferentes partes de las astas de venado de las Pampas y las técnicas involucradas en la obtención de las formas base y su posterior formatización o uso en el centro de la subregión Pampa Húmeda de Argentina. Para llevarlo a cabo, se efectuó el análisis de los restos de cornamentas de dos sitios arqueológicos: Calera y Empalme Querandíes 1
(Figura 1), correspondientes al Holoceno Tardío (3500 a 500 AP). La elección de la muestra se basa en la gran cantidad de restos de astas recuperada en estos conjuntos, en relación con la mayoría de los sitios, en los cuales dichos especímenes suelen restringirse a uno o dos ítems. Por este motivo, constituyen una excelente oportunidad para comenzar a indagar acerca del uso de las cornamentas y sus técnicas de manufactura.

\section{Antecedentes}

\section{Las astas}

Una de las principales características de los cérvidos es que son los únicos mamíferos a los cuales les crecen cornamentas cada año. Estos elementos son caracteres sexuales secundarios que sirven para las luchas intra-sexuales (Goss 1983). Cada especie posee cornamentas con una forma particular, la cual puede variar con la edad (Brown 1980). Las astas son apéndices del cráneo que crecen en la gran mayoría de los machos de los cérvidos, a partir de pedicelos óseos. Estos son protuberancias permanentes del hueso frontal y suelen desarrollarse en la primavera del segundo año de vida del animal (Price et al. 2005). El comienzo de su formación coincide con la aparición del terciopelo o felpa, una piel que cubre la parte distal del pedicelo. Los pedicelos crecen por la estimulación del periostio que generan las hormonas masculinas (Goss 1983). Las astas son un producto de la depositación de minerales, transportados por el terciopelo, que es un tejido muy vascularizado.

El asta está formada por tejido conectivo y cartilaginoso, el cual se osifica por medio de un proceso de mineralización (Brown 1980). A medida que termina el proceso de crecimiento, el tejido esponjoso de los bordes de las astas es reemplazado por hueso compacto y la parte central se rellena con hueso esponjoso. Este último es poco denso, blando y débil, en tanto que el hueso compacto es más denso y fuerte (Demarais y Strickland 2011). El crecimiento de las astas comienza luego del volteo, en primavera, y continúa hasta el otoño, cuando se vuelve totalmente mineralizada y la felpa comienza a secarse y a exponer el hueso (Price et al. 2005). La felpa muere, se deshidrata y cae y también es eliminada por frotamiento contra la vegetación. La cornamenta se renueva anualmente y con el paso del tiempo, va aumentando su tamaño y sus ramificaciones. En total, el proceso de crecimiento del asta dura entre dos y tres meses y medio, en tanto que las astas mineralizadas y limpias permanecen en el animal unos ocho meses y medio (Brown 1980). 


\section{El venado de las Pampas}

El venado de las Pampas (Figura 2a) es un cérvido mediano para el que se registran cinco subespecies, de las cuales Ozotoceros bezoarticus celer es la que habita los pastizales pampeanos (Chébez y Johnson 1994; González et al. 2010). Su distribución fue muy amplia hasta mediados del siglo XIX y abarcaba el sudeste y centro de Brasil, el sudeste de Bolivia, Paraguay, Uruguay y el noreste y centro de Argentina (Merino 2003). Sin embargo, la caza y la modificación de los hábitats provocaron una reducción en las poblaciones de venados y en su distribución (Chébez et al. 2008). En el registro fósil se reconoce la especie para la región pampeana desde el Holoceno Temprano (Cione et al. 1999).

La altura del venado es de aproximadamente $70 \mathrm{~cm}$ en la cruz y su peso oscila entre los 30 y $40 \mathrm{~kg}$ (Chébez y Johnson 1994). Como la gran mayoría de los cérvidos, la principal diferencia sexual son las cornamentas en los machos y el tamaño ligeramente mayor que estos presentan con respecto a las hembras (Jackson 1987). La estructura social del venado de las Pampas es compleja; los grupos típicos incluyen entre cinco y 17 individuos, de ambos sexos y de diferentes edades. Estos animales se juntan y dispersan continuamente, a veces en parejas o individuos solitarios (González et al. 2010). Los machos suelen abandonar su territorio para formar harenes (Chébez y Johnson 1994). Las hembras dan a luz una sola cría, entre los meses de octubre y noviembre. Sus principales predadores son el puma y el jaguar, aunque los zorros y otros carnívoros pueden llegar a predar sobre los cervatos (González et al. 2010; Jackson 1987).

Las cornamentas de los venados de las Pampas no exceden los $30 \mathrm{~cm}$ de longitud. Estas tienen tres puntas, una de ellas es simple, dirigida hacia adelante (garceta luchadora), en tanto que la rama, hacia atrás, se divide en dos puntas (garceta anterior y posterior) (Chébez y Johnson 1994) (Figura 2b). A partir de su madurez (12 meses), los machos adquieren una punta de la cornamenta por año, hasta llegar a tres de cada lado (Chébez y Johnson 1994). Las cornamentas caen generalmente entre agosto y septiembre, al final del invierno. La regeneración comienza casi inmediatamente, entre octubre y noviembre (Jackson 1986). Las astas tienen felpa durante unos 30 a 45 días y están totalmente desarrolladas y limpias (sin felpa) a principios del verano, para el período de la brama, que tiene lugar entre febrero y mayo (González et al. 2010; Jackson y Langguth 1987) (Figura 2c).

\section{Las Muestras Arqueológicas}

Las muestras proceden de dos sitios arqueológicos a cielo abierto: Empalme Querandíes 1 y Calera. Estos se localizan en el límite entre el Sistema Serrano de Tandilia occidental y la Llanura Interserrana, en el centro de la subregión Pampa Húmeda. Ambos corresponden a ocupaciones de cazadores-recolectores del Holoceno Tardío.
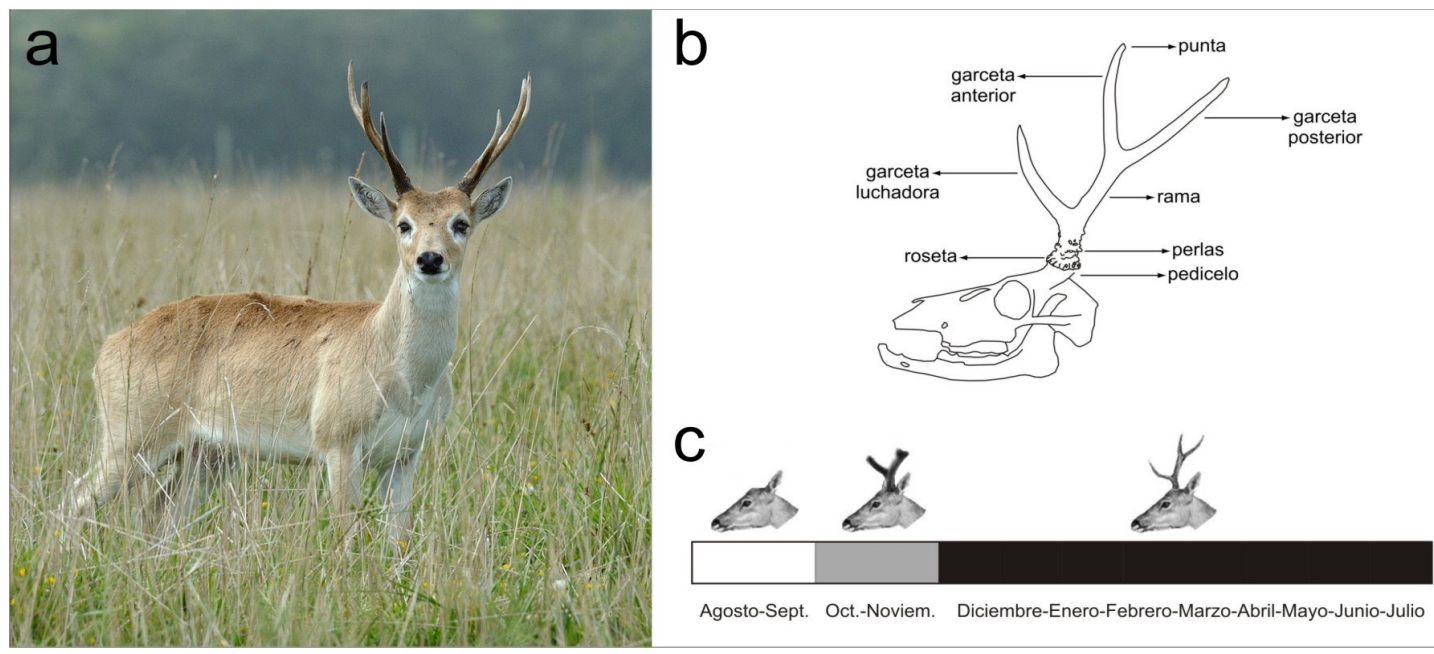

Figura 2. (a) Venado de las Pampas. Tomado de https://es.wikipedia.org/wiki/Ozotoceros_bezoarticus; (b) esquema de un cráneo con cornamenta y nombre de cada una de sus partes; (c) esquema del ciclo de las astas.

(a) Pampas deer. Taken from https://es.wikipedia.org/wiki/Ozotoceros_bezoarticus; (b) sketch of the skull with the name of each part of the antler; (c) sketch of the antler cycle. 
El sitio Empalme Querandíes 1 (en adelante $\mathrm{EQ} 1,37^{\circ} 00^{\prime}, 22$ 'S, 60 22' 39' O, Figura 1) fue excavado $\left(8 \mathrm{~m}^{2}\right)$ por Messineo y la autora entre los años 2010 y 2011. Los análisis efectuados indican que corresponde a un campamento de actividades múltiples, ocupado por cazadores-recolectores durante diferentes momentos del Holoceno Tardío. Se cuenta con tres fechados radiocarbónicos obtenidos sobre huesos de guanaco, que arrojaron edades de $3095 \pm 50$ AP, 2816 \pm 49 AP y $2052 \pm 62$ AP (Messineo et al. 2013). El análisis faunístico del sitio mostró el consumo de varios taxones, principalmente guanaco y en menor medida venado de las Pampas, vizcacha (Lagostomus maximus), ñandú (Rhea americana) y distintas especies de armadillos. En cuanto a la tecnología lítica, los artefactos más abundantes fueron confeccionados en ortocuarcita y ftanita. Entre estos se destacan los artefactos compuestos, piezas de retoque sumario, raederas, cuchillos y raspadores. También se registra una gran cantidad de tiestos cerámicos lisos y decorados (Colantonio et al. 2016; Messineo et al. 2013).

El sitio Calera ( $36^{\circ} 58^{\prime} 56^{\prime \prime} \mathrm{S}, 60^{\circ} 14^{\prime} 24^{\prime \prime} \mathrm{O}$, Figura 1) fue excavado en pocos días en el año 2005 por Politis, Messineo y Kaufmann, durante tareas de arqueología de rescate (Messineo y Politis 2007; Politis et al. 2005). Los materiales se hallaban dispuestos en cuatro depresiones en forma de cubetas, que ocupaban una superficie aproximada de $15 \mathrm{~m}^{2}$ y comprendían gran cantidad de restos óseos, artefactos líticos, fragmentos cerámicos y pigmentos minerales, entre otros. Entre los artefactos líticos predominan las raederas, raspadores y lascas con filos naturales con rastros complementarios. Por otra parte, las especies presentes incluyen una importante variedad de taxones (especialmente mamíferos y aves), aunque predomina ampliamente el guanaco. Se realizaron nueve fechados radiocarbónicos, ocho de ellos sobre colágeno de hueso, que arrojaron edades de $1748 \pm 42$ AP, $2232 \pm 55$ AP, $3390 \pm 170$ AP, $3160 \pm 320$ AP, $2772 \pm 51$ AP, $3008 \pm 44$ AP, $3005 \pm 66$ AP y $2075 \pm 44$ AP. Finalmente, un fechado sobre Gastropoda indicó una edad de 1500 \pm 58 AP (Messineo y Politis 2007; Politis et al. 2005). Sobre la base de distintas líneas de evidencia se propuso que Calera se formó como producto de la depositación diacrónica de ofrendas y/o basura ceremonial, efectuada durante rituales o festines ocurridos en las inmediaciones del sitio (Álvarez 2009; Messineo y Politis 2007; Politis et al. 2005; Scheifler 2014; entre otros).

\section{Materiales y Métodos}

La determinación de los especímenes de asta se realizó mediante la comparación con muestras modernas alojadas en la Unidad Ejecutora INCUAPA-
CONICET (Facultad de Ciencias Sociales, Universidad Nacional del Centro de la Provincia de Buenos Aires). Se dividió a la cornamenta en puntas -distinguiendo cuando fue posible en garceta luchadora, anterior, posterior o indeterminada-, rosetas y ramas. Además, si bien no son parte de la cornamenta, se incluyeron los pedicelos, cuando estuvieron presentes. En el caso de las rosetas que no estaban adheridas al cráneo, se determinó si correspondían a astas de volteo por la presencia de la línea de abscisión, que es el área donde el pedicelo se encuentra con el asta (Steve Demarais comunicación personal 2018). Se calculó el Número Mínimo de Individuos y Elementos (MNI, MNE, Binford 1978; Klein y Cruz Uribe 1984) para la cornamenta, asumiendo que se trataba de individuos adultos (tres puntas), para no sobredimensionar el número de astas.

Los especímenes se observaron a ojo desnudo y con lupa estereoscópica y luz incidente. Con respecto a las marcas, se consideraron los criterios de Jin y Shipman (2010). Estas autoras caracterizaron una serie de variables a partir del análisis de una colección moderna de cornamentas de venado de cola blanca (Odocoileus virginianus). Estas consisten en modificaciones que se producen de manera natural cuando los cérvidos frotan sus astas contra el follaje para remover el terciopelo seco o en las competencias con otros machos. La mayoría de las marcas aparecen en las puntas de las cornamentas y son: los arañazos o rayaduras (scratches), las superficies pulidas y las puntas biseladas. Las rayaduras son marcas poco profundas, numerosas y orientadas azarosamente. En general, el lado externo de la superficie suele estar más rayado y la mayoría de estas marcas se ubican en la mitad superior. Las superficies pulidas se registran en todas las cornamentas, incluso en algunas con felpa, lo cual indica que este rasgo responde a la morfología natural del asta y no sólo al contacto con objetos. En cuanto a las puntas biseladas, esto ocurre cuando los extremos del asta se rompen por causas naturales. En general estas aparecen también pulidas (Jin y Shipman 2010).

Se consideró la presencia de otro tipo de marcas en la superficie cortical, como por ejemplo huellas de corte, negativos de lascados o abrasión (Diakowski 2011). En el caso de los negativos de lascado, estos no son tan evidentes como en los huesos y en general se manifiestan como depresiones de fondo rugoso (Baumann y Mary 2013). Asimismo, se registró la existencia de alteración térmica, así como de variables tafonómicas, cuando estuvieron presentes. En relación con la meteorización, solo se identificó si el espécimen la presentaba o no, pero no se emplearon estadíos debido a que el asta tiene una estructura particular y se desconoce si se deteriora de la misma forma que los demás huesos del esqueleto. 
Con respecto a las fracturas, tampoco se cuenta con criterios diagnósticos que permitan identificar claramente el origen de las mismas en las cornamentas. Una vez separada del cráneo, el asta se comporta como un hueso seco. Averbouh y Pétillon (2011) lograron distinguir algunos criterios que les permitieron diferenciar fracturas secas de frescas. De acuerdo con estos autores, en las fracturas en estado seco el plano de fractura y la parte externa del asta forman un ángulo amplio (en general cercano a $\operatorname{los} 90^{\circ}$ ) y la superficie de fractura puede parecer irregular y áspera. Por el contrario, en el caso de las fracturas en estado fresco el plano y la sección externa del asta forman un ángulo estrecho (en general cercano a los $45^{\circ}$ ) y la superficie de fractura muestra la estructura fibrosa del material. Por otra parte, también pueden registrarse fracturas modernas, producidas durante el hallazgo o excavación del sitio.

\section{Resultados}

\section{Identificación Anatómica}

Se analizó un total de 38 especímenes determinados como fragmentos de astas o pedicelos de venado de las Pampas. Treintaiuno de ellos procede del sitio Calera y siete de EQ1. En la Tabla 1 se observan estos datos considerando todas las combinaciones en que se presentaron los restos. Por otra parte, en la Tabla 2 se muestra la recomposición y la estimación del MNE. Dicha medida indica que el número mínimo de elementos asciende a tres en EQ1 y a ocho en Calera, en tanto que el número mínimo de individuos es de dos en $\mathrm{EQ} 1$, sobre la base de dos rosetas izquierdas, y de ocho en Calera, a partir de la recomposición de las rosetas derechas, izquierdas e indeterminadas. Es necesario aclarar que uno de los elementos de EQ1 corresponde a un cráneo con las bases de las dos rosetas, con lo cual si bien se trata de un espécimen, en las tablas se ingresó como dos, ya que se consideró a cada cornamenta como un elemento diferente.

Tabla 1. Partes de la cornamenta representadas en EQ1 y Calera. Parts of the antler represented in EQ1 and Calera.

\begin{tabular}{ccc}
\hline Parte & EQ1 & Calera \\
\hline Garceta indeterminada & 2 & 10 \\
Garceta luchadora & 2 & 1 \\
Roseta con garceta luchadora & 1 & 1 \\
$\begin{array}{c}\text { Fragmento de cráneo con roseta } \\
\text { (asta no volteada) }\end{array}$ & 2 & 3 \\
Pedicelo & & \\
Roseta & 0 & 2 \\
Roseta con garceta luchadora y rama & 0 & 1 \\
Rama & 0 & 2 \\
Fragmento indeterminado & 1 & 2 \\
\hline
\end{tabular}

Tabla 2. Número mínimo de cornamentas en EQ1 y Calera.

\begin{tabular}{ccccccccc}
\multicolumn{1}{c}{ Minimum number of antlers in EQ1 and Calera. } \\
& \multicolumn{1}{c}{ EQ1 } & \multicolumn{5}{c}{ Calera } \\
& Izq & Der & Indet & MNE & Izq & Der & Indet & MNE \\
\hline Pedicelo & 1 & 1 & - & 2 & 3 & 2 & - & 3 \\
Roseta & 2 & 1 & - & 3 & 3 & 5 & 7 & 8 \\
Garceta luchadora & 1 & - & 2 & 2 & - & 2 & 1 & 2 \\
Garceta indeterminada & - & - & 2 & 1 & - & & 10 & 4 \\
Rama & - & - & - & - & - & 1 & 2 & 2 \\
Fragmento indeterminado & - & - & 1 & 1 & - & - & 2 & 1 \\
\hline
\end{tabular}




\section{Modificaciones en las puntas}

Se determinó un número de 17 especímenes correspondientes a puntas, procedentes de ambos sitios. En el 100\% de ellas se relevaron superficies pulidas y rayaduras (Figura 3). Asimismo, tres presentaban las puntas biseladas (17,65\%, Figura 3a) y en cinco de los restos $(29,41 \%)$ no pudo determinarse porque la zona distal estaba fracturada.

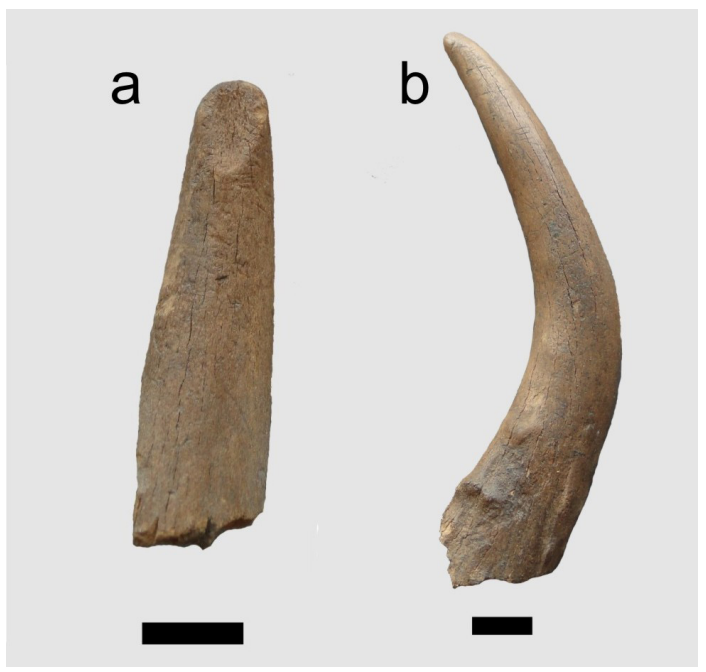

Figura 3. (a) Fragmento de garceta con rayaduras y punta biselada; (b) garceta luchadora con superficie pulida y rayaduras. Escala=1 $\mathrm{cm}$.

(a) Fragment of tine with scratches and beveled tip; (b) Brow tine with polished surface and scratches. Scale $=1 \mathrm{~cm}$.

En el caso de las fracturas del sector proximal de la punta, dos de ellas estaban unidas a las bases. En cuanto al resto, se registraron siete fracturas modernas $(46,66 \%)$, cuatro en estado seco $(26,67 \%)$ y cuatro en estado fresco $(26,67 \%)$. Por otra parte, una de las puntas presentaba dos claras huellas de corte, transversales al eje. Finalmente, en nueve especímenes se relevó meteorización $(52,94 \%)$ y en ocho no $(47,06 \%)$.

\section{Modificaciones en las bases}

Se identificaron 19 bases de las cuales $12(63,16 \%)$ eran de volteo, cinco $(26,32 \%)$ correspondían a fragmentos del hueso frontal con la base de la roseta adherida y dos $(10,53 \%)$ eran pedicelos en estado de crecimiento. Ninguna de las bases presentaba superficies pulidas ni rayaduras. Durante el análisis se advirtió que cuatro de las bases presentaban la ausencia de todo el borde de la roseta y negativos de lascado en el perímetro de la misma (Figura 4). En estos casos se observan aun las perlas, pero la zona de la línea de abscisión está abradida.

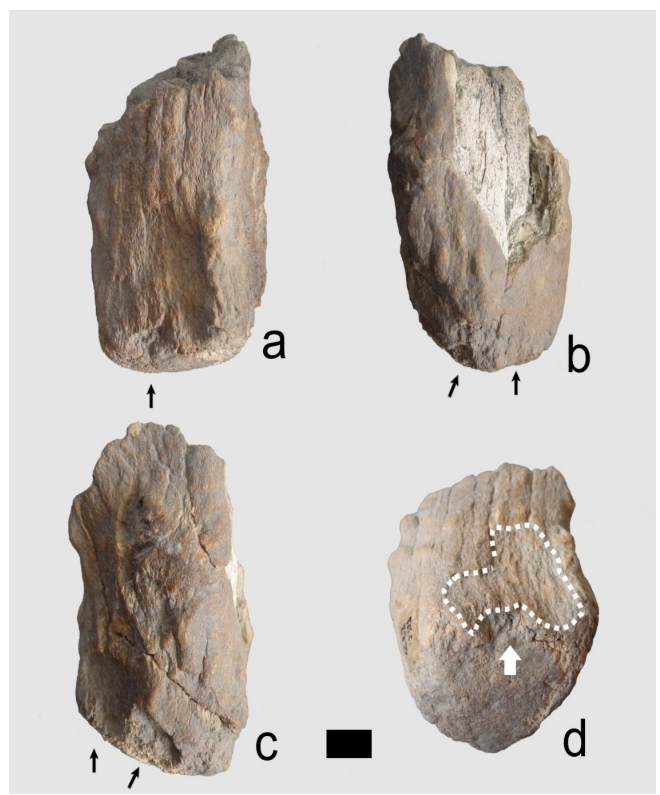

Figura 4. (a-c) Vistas de una roseta con ausencia de borde y negativos de lascado en su base. Las flechas señalas algunos negativos de lascado; (d) vista de uno de los negativos de lascado con el espécimen en posición oblicua. Escala $=1 \mathrm{~cm}$.

( $a-c)$ Views of a burr with the absence of the edge and flake negatives at the base. Arrows point to some of the flake negatives; $(d)$ view of one of the flake negatives at an oblique position. Scale $=1 \mathrm{~cm}$.

Por otra parte, en seis casos se registró además de los negativos de lascado y ausencia del borde, la abrasión de toda la superficie del espécimen, estando las perlas o bien ausentes o suavizadas (Figura 5). Además, una de estas presentaba numerosas huellas de corte profundas y en diferentes direcciones (Figura 6).

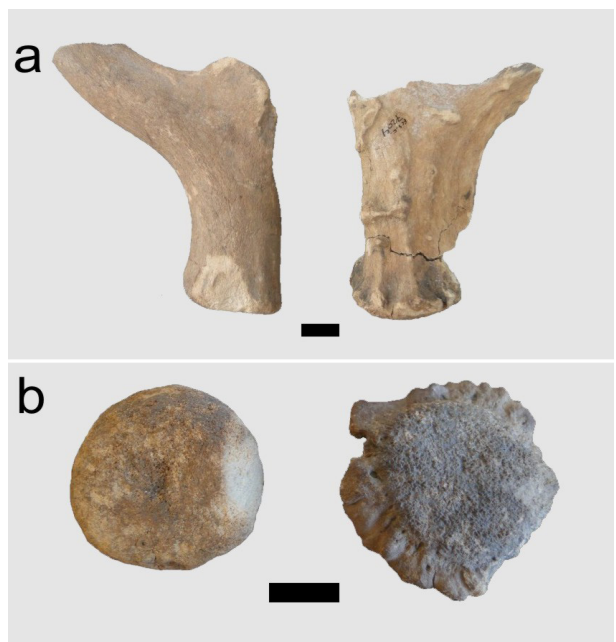

Figura 5. Comparación entre una roseta normal (derecha) y una abradida (izquierda): (a) vista lateral; (b) vista de la base. Escala $=1 \mathrm{~cm}$.

Comparison between a normal burr (right) and one with abrasion (left): (a) lateral view; (b) view from the base. Scale $=1 \mathrm{~cm}$. 


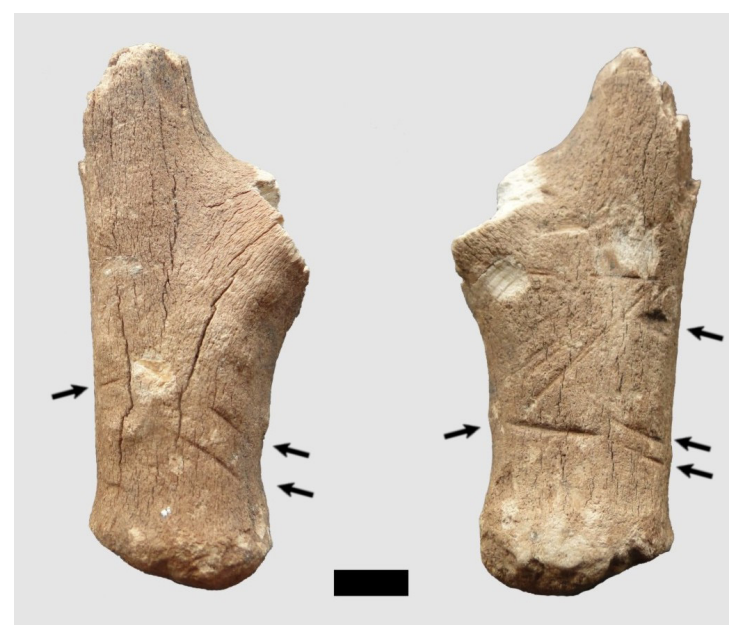

Figura 6. Especímen abradido y con ausencia del borde de la roseta. Las flechas señalan algunas de las huellas de corte reconocidas. Escala $=1 \mathrm{~cm}$.

Specimen with abrasion and with absence of the edge of the burr. Arrows point to some of the cut marks identified. Scale $=1 \mathrm{~cm}$.

Con respecto a los fragmentos de los especímenes con astas no volteadas se registró el caso del cráneo de EQ1, el cual posee marcas de machacado y alteración térmica en ambas cornamentas (Figura 7). A su vez, dos especímenes del sitio Calera presentan machacado, inmediatamente después de la base de la roseta, posiblemente para desprender el resto de la cornamenta. Además, en estos ítems también falta todo el borde de la roseta y esta zona exhibe negativos de lascado (Figura 8).

En el caso de las fracturas, nueve son modernas $(47,37 \%)$, cuatro en estado seco $(21,05 \%)$ y seis en estado fresco $(31,58 \%)$. El alto porcentaje de fracturas modernas se dio especialmente en el caso del sitio Calera, el cual se trataba de un contexto de rescate. Finalmente, 11 especímenes $(57,89 \%)$ no presentan meteorización y ocho sí $(42,11 \%)$.

\section{Discusión}

La presencia de restos de astas de venado de las Pampas en los sitios arqueológicos podría estar vinculada con diferentes fines. En el caso de las astas de volteo, la incorporación al sitio estaría relacionada con motivos tecnológicos, dado que estos elementos no tienen productos alimenticios. Los fragmentos de cráneos con astas adheridas pueden haber cumplido la doble función de haber sido explotado el animal

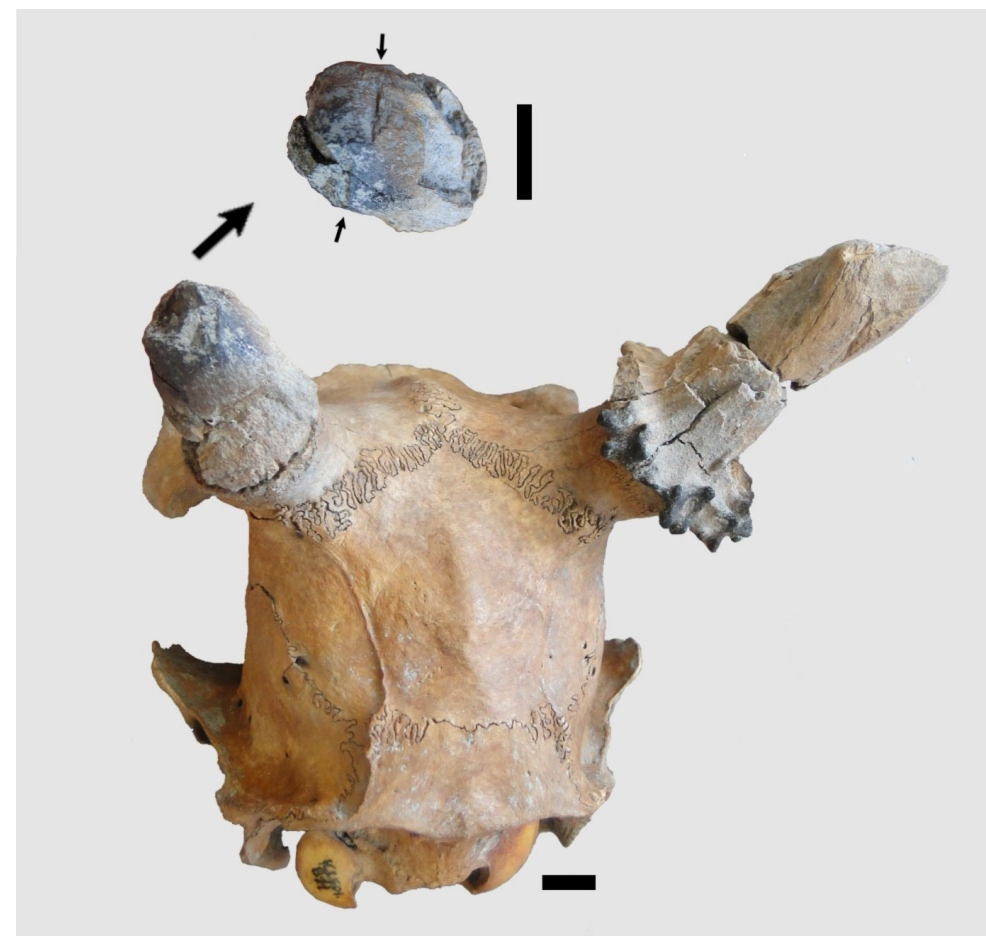

Figura 7. Cráneo de EQ1 con detalle de machacado en la base. Escala $=1 \mathrm{~cm}$.

Skull from EQ1 with detail of chopping in the base. Scale $=1 \mathrm{~cm}$. 


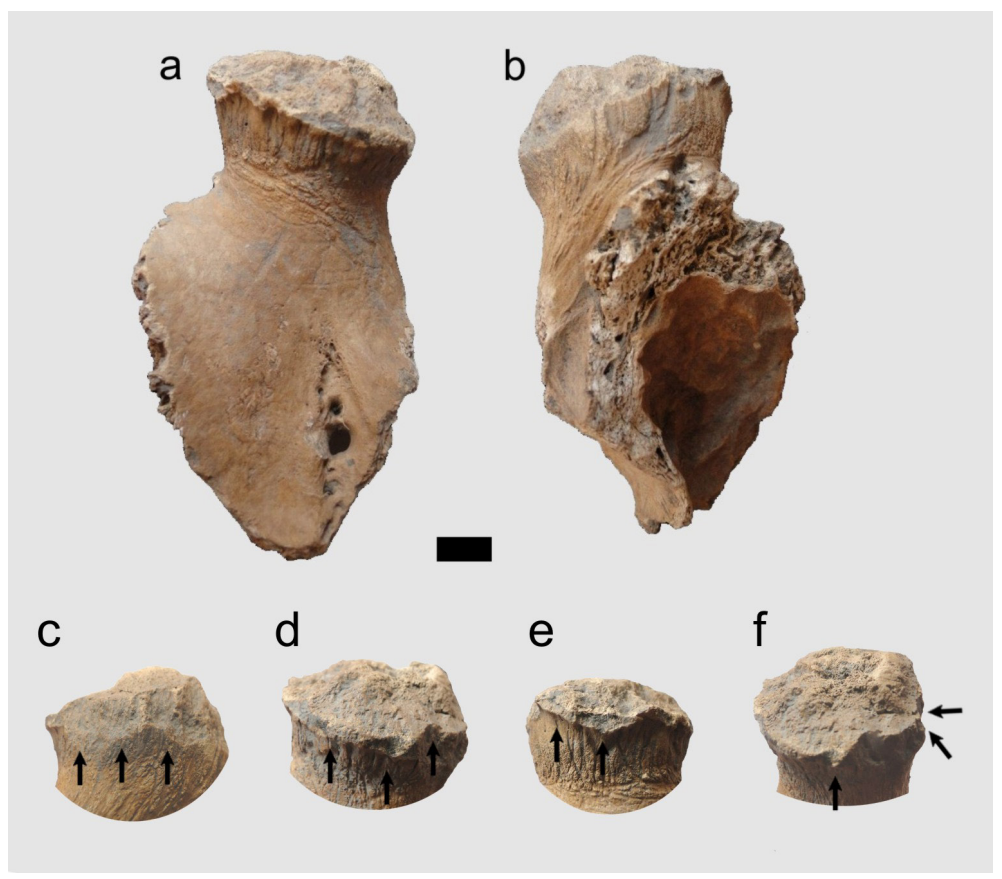

Figura 8. Fragmento de cráneo de Calera con base de roseta. (a-b) vista anterior y posterior; (c-f) diferentes vistas con detalle de negativos de lascado. Escala $=1 \mathrm{~cm}$.

Skull fragment from Calera with burr base. (a-b) anterior and posterior view; (c-f) different views with detail of flake negatives. Scale $=1 \mathrm{~cm}$.

para la subsistencia y la tecnología. En el caso de los pedicelos, probablemente serían consecuencia del aprovechamiento del animal para la subsistencia, aunque no se descarta el posible uso de la felpa con motivos medicinales, como se ha registrado en diferentes partes del mundo (Tuckwell 2003). En relación con este aspecto, existen datos acerca de que los cazadores Cherokee atribuían a la felpa la propiedad de atraer la buena suerte; en los sitios prehistóricos de estos grupos también se ha registrado la ocurrencia de garcetas de venado de cola blanca que funcionaron como amuletos de caza (Peres y Altman 2018).

Dado que el asta tiene un ciclo anual, el estado de la cornamenta constituye un indicador de estacionalidad. En relación con los materiales aquí analizados se registran, para Calera, tanto pedicelos como astas de momentos anteriores y posteriores al volteo, lo cual indicaría diferentes momentos del año para las ocupaciones. En el caso de los pedicelos, estos están presentes en los meses de octubre y noviembre, lo cual permite acotar la estacionalidad. Sin embargo, las astas de volteo e incluso aquellas adheridas al cráneo, podrían haber sido recolectadas en cualquier momento del año y por esto no necesariamente constituyen indicadores confiables de estacionalidad. En trabajos previos se construyó un perfil de mortalidad de guanacos para el sitio Calera. Coincidentemente con los datos de las astas, este indicó que si bien se registran individuos de diferentes edades, hay una importante cantidad de neonatos, los cuales nacen entre los meses de noviembre y diciembre (Álvarez 2012; Kaufmann y Álvarez 2007). Con respecto a EQ1, la cantidad de especímenes es menor, pero se registró la presencia de un asta de volteo y un cráneo con sus cornamentas bien desarrolladas, posiblemente de momentos cercanos a la muda. Si se considera que este último fue obtenido a partir de la caza, esto podría indicar ocupaciones durante el invierno, lo cual también es concordante con el perfil de mortalidad de guanaco, que muestra ocupaciones en diferentes momentos del año, algunos de ellos durante dicha estación (Álvarez 2012).

Considerando todos los especímenes en su conjunto, se observó la presencia de partes segmentadas del asta, divididas generalmente en garceta y roseta, aunque existe una mayor variedad de combinaciones. Además, se relevó la existencia de machacado en algunos sectores, principalmente en la zona de la roseta, con el probable objetivo de separar a esta del resto de la cornamenta. La presencia de fracturas en estado fresco también se condice con gestos vinculados con la división del elemento en porciones. Por esto se propone que la técnica de obtención de las formas base corresponde a lo que Averbouh y Pétillon (2011) denominaron como débitage por segmentación. Esta 
técnica consiste en la división del asta en segmentos para ser utilizados como formas base y se realiza de forma transversal, usualmente por medio de cortes, ranurado y/o machacado. Otras técnicas registradas para el Paleolítico europeo son el débitage por extracción, que consiste en obtener la parte externa del asta (sólo el tejido compacto) y se realiza de forma longitudinal, y el débitage por fracturación, por medio del cual se fractura la cornamenta por talla, para producir lascas (Averbouh y Pétillon 2011).

Una vez segmentada la cornamenta, probablemente por percusión directa, los productos obtenidos habrían sido las puntas y las rosetas (ya sea volteadas o no). En el caso de las ramas, estas fueron muy escasas y no se registraron fracturas en estado fresco $u$ otros rasgos que indiquen su utilización como instrumentos. Aunque no se descarta su uso, las mismas serían, posiblemente, desechos de la manufactura. Con respecto a las puntas, los rasgos identificados, correspondientes a superficies pulidas, puntas biseladas y rayaduras, se producen naturalmente en las cornamentas en vida de los animales y no permiten diferenciar este origen del antrópico (Jin y Shipman 2010; Olsen 1989). No obstante, esto no significa que no hayan sido aprovechadas. En este sentido, la presencia del cráneo de EQ1 con la base de las rosetas adheridas y marcas de machacado, sugiere la selección y posible utilización del resto de la cornamenta. Para el centro de la Pampa Húmeda, se propuso que algunas de las puntas podrían haber sido utilizadas como retocadores o para la perforación de cueros, pero recientemente se ha planteado que podrían haber estado vinculadas con labores textiles (Mazzanti y Puente 2015), aunque no se descartan los usos mencionados previamente.

En relación con las bases, se observó la presencia de negativos de lascado y abrasión, tanto en las rosetas de cornamentas de volteo, como en otras que aún permanecían adheridas al cráneo. Estas modificaciones no se producen en vida del animal. En este sentido, estas secciones constituirían partes diagnósticas del uso del asta con fines tecnológicos (Olsen 1989). Es posible que estas porciones hayan sido utilizadas como percutores blandos. Los negativos de lascado y la abrasión podrían haberse generado tanto por la formatización de estos elementos como por su uso posterior. La abrasión es una técnica que ha sido propuesta como parte de la formatización del artefacto, para la remoción de las perlas (Diakowski 2011). Por otra parte, experimentos con el uso de la base del asta de venado de cola blanca como percutor blando indican que la pérdida atricional de la superficie cortical, la compactación y la presencia de hoyuelos están entre los rasgos diagnósticos para identificar este tipo de herramientas (Bello et al. 2013). En cuanto a las huellas de corte profundas en uno de los especímenes, por el momento se desconoce su origen.
En las sierras centrales de Tandilia se registró la presencia de astas modificadas en un contexto de afloramiento y explotación de rocas. Se trata del sitio El Picadero (La Numancia, Tandil), donde fue recuperado un individuo de venado de las Pampas, con alto grado de completitud, que ha sido fechado en ca. 600 AP (Colombo 2012). Este venado presentaba sus dos cornamentas incompletas, con ambas rosetas con negativos de lascado en el sector proximal y ausencia del borde, lo que llevó a proponer su posible uso como percutores blandos (Colombo 2012). Por otra parte, en el marco de este trabajo se observaron los restos de asta del sitio Paso Otero 4 (partido de Necochea). Si bien muchas de ellas estaban fragmentadas, una que tenía mayor completitud presentaba negativos de lascado en la zona de la roseta. Este espécimen corresponde a los niveles superiores del sitio, asignados a una cronología de ca. 7700 a 4600 AP (Álvarez et al. 2013; Gutiérrez et al. 2011), con lo cual la evidencia directa del uso de esta tecnología relevada para el Holoceno Tardío se extiende al menos al Holoceno Medio. En este punto es importante destacar que el uso de percutores blandos a partir de la evidencia indirecta del estudio de los materiales líticos fue propuesto para la región pampeana desde momentos tan tempranos como la transición Pleistoceno-Holoceno (p.ej., Bonnat y Mazzanti 2015).

La presencia de una gran cantidad de rosetas con modificaciones en el sitio Calera (10 en total), las cuales probablemente funcionaron como "percutores blandos", podría estar vinculada con la funcionalidad del sitio, relacionada con las ofrendas ceremoniales. En este tipo de contextos suele ser usual el enterramiento intencional de elementos que están en los primeros estadios de reducción, los que no deberían haber sido descartados si se tiene en cuenta solo sus fines utilitarios (Politis et al. 2005). En este sentido, este contexto constituye una oportunidad única de hallar concentrados materiales que generalmente son descubiertos de manera ocasional. En relación con este punto, es probable que los instrumentos sobre asta hayan estado vinculados con una estrategia más conservada y por este motivo no sean tan frecuentes en el registro de los sitios arqueológicos de la región.

\section{Conclusión}

En este trabajo se analizaron los restos de astas de dos sitios arqueológicos, uno de los cuales presenta un registro excepcional, que permitió acceder al conocimiento de una tecnología que, si bien habría sido ampliamente utilizada, se recupera en escasas proporciones en los sitios arqueológicos. A partir de esto se pudo determinar que se aprovecharon tanto astas de volteo (recolectadas) como otras aun adheridas al cráneo. Con respecto a la técnica de obtención de las 
formas base, la misma habría sido la de débitage por segmentación, posiblemente por percusión directa. En el caso de los segmentos utilizados, si bien es probable que todas las partes hayan sido aprovechadas, en ausencia de otros rasgos diagnósticos como marcas y fracturas, las puntas no constituirían los elementos más diagnósticos del uso de las cornamentas como tecnología. Por el contrario, las bases modificadas serían las partes más fácilmente reconocibles. Esto había sido notado previamente por Olsen (1989). Sin embargo, en general, en el centro de la Pampa Húmeda se ha prestado mayor atención a las garcetas, que se registran con más frecuencia que las bases. Esto podría responder a que estás últimas hayan tenido menores chances de preservarse en el registro fósil, debido a que están constituidas en gran medida por tejido esponjoso.

Se propone que en futuras investigaciones o en la revisión de colecciones arqueológicas, debería hacerse un mayor foco en las rosetas, cuando estas están presentes en los sitios, con el objetivo de poder evaluar cuán frecuente y extendido fue su uso como percutores en el pasado. Finalmente, se espera que en análisis próximos se puedan realizar estudios funcionales que permitan comparar los rasgos a nivel microscópico con los experimentos desarrollados por algunos autores (Bello et al. 2013) o bien, con una colección generada localmente.

Agradecimientos. A Marcelo Pauluzzi y al profesor Guillermo Demo, quienes generosamente me cedieron materiales para poder llevar a cabo este trabajo. A Mariano Merino, Rodolfo Ungerfeld y Steve Demarais, que respondieron a mis consultas acerca de algunos de los especímenes analizados. A Pablo Messineo, Guillermo Heider y Cristian Kaufmann por la lectura crítica del manuscrito. A los proyectos PICT 20150235, 2014-2070 y PIP 0414 por el financiamiento. Al INCUAPA-CONICET y la Facultad de Ciencias Sociales, Olavarría, por el apoyo institucional. A tres evaluadores anónimos que contribuyeron a mejorar este trabajo con sus sugerencias.

\section{Referencias Citadas}

Acosta, A., N. Buc y L. Mucciolo 2014. Elementos óseos con aserrado perimetral: el caso del Humedal del río Paraná Inferior. Revista Chilena de Antropología 30 (2):17-23.

Álvarez, M.C. 2009. Análisis de los restos faunísticos del sitio Calera (Sierras Bayas, partido de Olavarría). Un aporte a su funcionalidad a través del estudio de los mamíferos. Relaciones de la Sociedad Argentina de Antropología 34:29-51.

Álvarez, M.C. 2012. Análisis Zooarqueológicos en el Sudeste de la Región Pampeana. Patrones de Subsistencia Durante el Holoceno Tardío. Tesis Doctoral. Facultad de Ciencias Sociales, Universidad Nacional del Centro de la Provincia de Buenos Aires, Buenos Aires, Olavarría.

Álvarez, M.C., A. Alcaraz, M. Gutiérrez y G. Martínez 2013. Análisis zooarqueológico del sitio Paso Otero 4 (partido de Necochea). Aportes a la discusión de modelos de subsistencia de la región pampeana. Intersecciones en Antropología 14 (2):383-398.

Averbouh, A. y J.M. Pétillon 2011. Identification of "debitage by fracturation" on reindeer antler: Case study of the Badegoulian levels at the Cuzoul de Vers (Lot, France). En Written in Bones. Studies on Technological and Social Context of Past Faunal Skeletal Remains, editado por J. Baron y B. Kufel-Diakowska, pp. 41-52. Uniwersytet Wroclawski, Instytut Archeologii, Wroclaw.

Baumann, M. y S. Maury 2013. Ideas no longer written in antler. Journal of Archaeological Science 40:601-614.

Bello, S., S. Parfitt, I. De Groote y G. Kennaway 2013. Investigating experimental knapping damage on an antler hammer: a pilot-study using high-resolution imaging and analytical techniques. Journal of Archaeological Science 40:4528-4537.

Bello, S., G. Delbarre, I. De Groote y S. Parfitt 2016. A newly discovered antler Flint-knapping hammer and the question of their rarity in the Palaeolithic archaeological record: Reality or bias? Quaternary International 403:101-117.
Binford, L. 1978. Nunamiut Ethnoarchaeology. Academic Press, Nueva York.

Bonnat, F. y D. Mazzanti 2015. Análisis de la tecnología lítica de las ocupaciones humanas efímeras durante la transición PleistocenoHoloceno: el caso de Cueva La Brava (Buenos Aires, Argentina). Intersecciones en Antropología 16 (1):287-300.

Bonomo, M. 2013. Reanálisis de la colección de Samuel Lothrop procedente del Delta del Paraná. Relaciones de la Sociedad Argentina de Antropología 38 (1):169-198.

Bonomo, M. y D.C. León 2010. Un contexto arqueológico en posición estratigráfica en los médanos litorales. El sitio Alfar (Pdo. de General Pueyrredón, Pcia. De Buenos Aires). En Mamul Mapü: Pasado y Presente desde la Arqueología Pampeana, editado por M. Berón, L. Luna, M. Bonomo, C. Montalvo, C. Aranda y M. Carrera Aizpitarte, Tomo 2, pp. 29-45. Libros del Espinillo, Ayacucho.

Brown, C. 1980. An Evaluation of Selection Factors for Antler Growth in Red Deer (Cervus elaphus). Tesis Doctoral. Lincoln University, Lincoln.

Buc, N. y D. Loponte 2007. Bone tool types and microwear patterns: some examples from the Pampa region, South America. En Bones as Tools: Current Methods and Interpretations in Worked Bone Studies, editado por C. Gates Saint Pierre y R. Walker, pp. 143-157. British Archaeological Report, Archaeopress, Oxford.

Chébez, J.C. y A. Johnson 1994. Venado de las Pampas. En Los que se Van: Especies Argentinas en Peligro, editado por J.C. Chébez, pp. 298-311. Editorial Albatros, Buenos Aires.

Chébez, J.C., A. Johnson y A.A. Pautasso 2008. Venado de las Pampas. En Los que se Van. Fauna Argentina Amenazada. Tomo 3, editado por J.C. Chébez, pp. 224-242. Editorial Albatros, Buenos Aires.

Cione, A.L., E.P. Tonni, M. Bond, A. Carlini, U. Pardiñas, G. Scillato Yané, D. Verzi y M.G. Vucetich 1999. Occurrence charts 
of Pleistocene mammals in the Pampean area, eastern Argentina. Quaternary in South America and Antarctic Peninsula 12:53-60.

Colantonio, M.J., N. Pal y P. Messineo 2016. Análisis de las prácticas de producción y uso de los materiales líticos del sitio Empalme Querandíes 1 (cuenca superior del arroyo Tapalqué, Región Pampeana). Arqueología 22 (2):243-267.

Colombo, M. 2012. Los Cazadores y Recolectores Pampeanos y sus Rocas. La Obtención de Materias Primas Líticas Vista desde las Canteras Arqueológicas del Centro de Tandilia. Tesis Doctoral. Facultad de Ciencias Naturales y Museo, Universidad nacional de La Plata, La Plata.

Currey, J. 1984. The Mechanical Adaptations of Bones. Princeton University Press, Princeton.

Demarais, S. y B.K. Strickland 2011. Antlers. En Biology and Management of White-tailed Deer, editado por D.G. Hewitt, pp. 107-145. CRC Press, Boca Raton.

Diakowski, M. 2011. Bone and antler artefacts from Pobiel 10, Lower Silesia, Poland. Are they really Mesolithic? En Written in Bones. Studies on Technological and Social Context of Past Faunal Skeletal Remains, editado por J. Baron y B. Kufel-Diakowska, pp. 93-116. Uniwersytet Wroclawski, Wroclaw.

Escosteguy, P., M. Salemme y M. González 2017. Tecnología ósea en la Depresión del Río Salado (provincia de Buenos Aires). Arqueología 23 (3):65-90.

Frontini, R., M. Álvarez, M. Salemme, R. Vecchi y C. Bayón 2017. Nuevas investigaciones sobre la tecnología ósea en el sudoeste de la provincia de Buenos Aires. Procesos de manufactura y uso Arqueología 23 (2):137-146.

González S., M. Cossé, F. Góss Braga, A.R. Vila, M. Merino, C. Dellafiore, J.L. Cartes, L. Maffei y M.G. Dixon 2010. Pampas deer Ozotoceros bezoarticus (Linnaeus 1758). En Neotropical Cervidology: Biology and Medicine of Latin American Deer, editado por J.M. Duarta y S. González, pp. 119-132. Funep/IUCN, Jaboticabal.

Goss, R. 1983. Deer Antlers: Regeneration, Function and Evolution. Academic Press, Nueva York.

Guthrie, D. 1983. Osseous projectile point: Biological considerations affecting raw material selection and design among paleolithic and paleoindian peoples, animals and archaeology: 1. En Hunters and their Prey, editado por J. Clutton-Brock y C. Grigson, pp. 274-294. BAR International Series 163, Oxford.

Gutiérrez, M., G. Martínez, H. Luchsinger, S. Grill, A. Zucol, G. Hassan, P. Barros, C. Kaufmann y M.C. Álvarez 2011. Paleoenvironments in the Paso Otero locality during late PleistoceneHolocene (pampean region, Argentina): an interdisciplinary approach. Quaternary International 245:7-47.

Jackson, J.E. 1986. Antler cycle in Pampas deer (Ozotoceros bezoarticus) from San Luis, Argentina. Journal of Mammalogy 67 (1):175-176.

Jackson, J.E. 1987. Ozotoceros bezoarticus. Mammalian Species 295:1-5.

Jackson, J.E. y A. Langguth 1987. Ecology and status of the pampas deer in the Argentinian pampa and Uruguay. En Biology and Managment of the Cervidae, editado por C.M. Wemmer, pp. 402-409. Smithsonian Inst. Press, Washington DC.

Jin, J. y P. Shipman 2010. Documenting natural wear on antlers: A first step in identifying use-wear on purported antler tools Quaternary International 211:91-102.
Kaufmann, C. y M.C. Álvarez 2007. La arqueofauna del sitio Calera (Sierras Bayas, región pampeana): un abordaje a los aspectos rituales del descarte de huesos de animales. En Arqueología en las Pampas, editado por C. Bayón, A. Pupio, M. González, N. Flegenheimer y M. Frère, pp. 745-764. Sociedad Argentina de Antropología, Buenos Aires.

Klein, R. y K. Cruz-Uribe 1984. The Analysis of Animal Bones from Archaeological Sites. The University of Chicago Press, Chicago.

Martínez, G. y M. Gutiérrez 2004. Tendencias en la explotación humana de la fauna durante el Pleistoceno final y Holoceno en la Región Pampeana (Argentina). En Zooarchaeology of South America, editado por G. Mengoni Goñalons, pp. 81-98. BAR International Series 1298, Oxford.

Martínez, G., P. Messineo, E. Piñeyro, C. Kaufmann y P. Barros 2001. Análisis preliminar de la estructura faunística del sitio Paso Otero 3 (Pdo. de Necochea, Pcia. de Bs. As., Argentina). En Arqueología Uruguaya Hacia el Fin del Milenio. IX Congreso Nacional de Arqueología, pp. 505-520. Bastión del Carmen, Colonia de Sacramento.

Massigoge, A. 2009. Arqueología de los Cazadores-Recolectores del Sudeste de la Región Pampeana: Una Perspectiva Tafonómica. Tesis Doctoral. Facultad de Ciencias Naturales y Museo, Universidad Nacional de La Plata, La Plata.

Mazzanti, D. y F. Valverde 2001. Artefactos sobre hueso, asta y valva. En Cueva Tixi: Cazadores y Recolectores de las Sierras de Tandilia Oriental, editado por D. Mazzanti y C. Quintana, pp. 157180. Laboratorio de Arqueología, Universidad Nacional de Mar del Plata, Mar del Plata.

Mazzanti, D. y V. Puente 2015. La producción textil como actividad doméstica de los cazadores-recolectores prehispánicos en la región pampeana, Argentina. Intersecciones en Antropología 16 (1):131144

Merino, M. 2003. Dieta y Uso de Hábitat del Venado de las Pampas, Ozotoceros bezoarticus celer, Cabrera 1943 (MammaliaCervidae) en la Zona Costera de Bahía Sanborombón, Buenos Aires, Argentina. Implicancias para su conservación. Tesis Doctoral. Facultad de Ciencias Naturales y Museo, Universidad Nacional de La Plata, La Plata.

Messineo, P. y G. Politis 2007. El sitio Calera. Un depósito ritual en las Sierras Bayas (sector noroccidental de Tandilia). En Arqueología en las Pampas, volumen 2, editado por C. Bayón, I. González, A. Pupio, N. Flegenheimer y M. Frère, pp. 697-720. Sociedad Argentina de Antropología, Buenos Aires.

Messineo, P., M.C. Álvarez, C. Favier Dubois, P. Steffan y M. Colantonio 2013. Estado de avance de las investigaciones arqueológicas en el sitio Empalme Querandíes 1 (centro de la subregión Pampa Húmeda, provincia de Buenos Aires). Comechingonia 17 (1):123-148.

Nami, H. y V.G. Scheinsohn 1997. Use-wear patterns on bone experimental flakers: A preliminary report. En Proceedings of the 1993 Bone Modification Conference, Hot Springs, South Dakota, editado por L.A. Hannus, L. Rossum y R.P. Winhan, pp. 256-264. Archaeology Laboratory, Agusana College, Sioux Falls, South Dakota.

Olsen, S. 1989. On distinguishing natural from cultural damage on archaeological antler. Journal of Archaeological Science 16:125-135.

Peres, T. y H. Altman 2018. The magic of improbable appendages: Deer antler objects in the archaeological record of the American South. Journal of Archaeological Science: Reports. En prensa. 
Politis, G. 2008. The Pampas and Campos of South America. En Handbook of South American Archaeology, editado por $\mathrm{H}$. Silverman y W. Isbell, pp. 235-260. Springer, Nueva York.

Politis, G. y M. Bonomo 2015. Una revisión del Túmulo de Campana. Relaciones de la Sociedad Argentina de Antropología 40 (1):149-181.

Politis, G., P. Messineo, C. Kaufmann, P. Barros, M.C. Álvarez, V. Di Prado y R. Scalise 2005. Persistencia ritual entre cazadoresrecolectores de la llanura pampeana. Boletín de Arqueología PUCP 9:67-90.

Politis, G., L. Prates, M. Merino y M. Tognelli 2011. Distribution parameters of guanaco (Lama guanicoe), pampas deer (Ozotoceros bezoarticus) and marsh deer (Blastocerus dichotomus) in Central Argentina: archaeological and paleoenvironmental implications. Journal of Archaeological Science 38 (7):14051416.
Price J.S., S. Allen y C. Faucheux 2005. Deer antlers: a zoological curiosity or the key to understanding organ regeneration in mammals? Journal of Anatomy 207 (5):603-618.

Salemme, M. 2014. Zooarqueología y paleoambientes. En Estado Actual de las Investigaciones en el Sitio Arqueológico Arroyo Seco 2, editado por G. Politis, M. Gutiérrez y C. Scabuzzo, pp. 6796. Universidad Nacional del Centro de la Provincia de Buenos Aires, Buenos Aires.

Scheifler, N. 2014. Zooarqueología de los pequeños vertebrados del sitio Calera (cuenca superior del arroyo Tapalqué, provincia de Buenos Aires). Aprovechamiento humano, depredación por aves rapaces y acción hídrica. Relaciones de la Sociedad Argentina de Antropología 39 (1):145-173.

Tuckwell, C. 2003. Velvet Antler. A Summary of the Literatura on Health Benefits. Rural Industries Research and Development Corporation, Kingston. 
